Check for updates

Cite this: RSC Adv., 2019, 9, 21233

\title{
Synthesis and characterization of amphiphilic branched silica derivatives associated with oligomeric medium
}

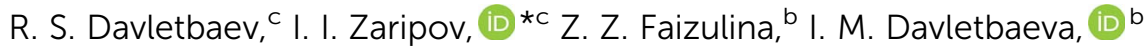 \\ D. S. Domrachova ${ }^{a}$ and A. M. Gumerov ${ }^{b}$
}

\begin{abstract}
Amphiphilic branched silica derivatives associated with oligomeric medium (ASiP) were obtained using tetraethoxysilane, polyoxyethylene glycol and low molecular weight polydimethylsiloxane. The creation of a silica core was based on tetraethoxysilane hydrolysis and condensation reactions by using water and a potassium diethylene glycolate as the catalyst. These reactions proceeded with the sequential participation of polyoxyethylene glycol and polydimethylsiloxane in parallel transetherification reactions. Microporous polymer film based on 2,4-toluene diisocyanate and block copolymers of propylene and ethylene oxides with terminal potassium-alcoholate groups were modified by ASiP. It has been shown that ASiP at the phase interface between thermodynamically incompatible macrochains performs the function of a link. It leads to a significant increase of intermolecular interactions and the supramolecular organization of the modified microporous polymers.
\end{abstract}

Received 16th May 2019

Accepted 27th June 2019

DOI: $10.1039 / c 9 r a 03683 k$

rsc.li/rsc-advances

chemical and physical properties. Amphiphilic macromolecular brushes are copolymers with narrowly dispersed side chains grafted to the main chain..$^{3-13}$ The properties of polymer brushes can be changed in a wide range compared with similar properties of linear polymers. The main design elements here are the length regulation of the main and side chains, the density of grafting, the chemical nature and architecture of the side chains. All these parameters are determined by the method of polymer brushes synthesis. Usually, there are two different approaches to the synthesis of macromolecular brushes. The first is a chemical method. In this case, a brush is grafted to a certain surface by terminal functional groups. Thus, some regularly branched polymers and flat brushes can be obtained, in which the chains are grafted to a flat surface. Spherically convex brushes can originate from star-shaped macromolecules that contain a large number of branches, and cylindrical convex brushes are obtained from comb-shaped polymers. The second way to form brushes is based on the ability of macromolecules to self-organize or self-assemble. A prerequisite for this is the presence of various groups in macromolecules. In simple cases, the formation of two-component copolymers is sufficient for these purposes, and the driving force of self-organization is intermolecular interaction. There are limitations of synthesis and certain advantages in molecular structures for each approach and method of obtaining macromolecular brushes. Important achieved parameters of synthesized macromolecular brushes are the grafting density, chemical composition, size, morphology, uniformity, length and distribution of the main and side chains. Asymmetric macromolecular brushes with polyethylene oxide and hydrocarbon side chains in polar 
solvents are assembled into micellar structure, whose crowns consist of polyoxyethylene side chains. The biggest part of researches in the field of amphiphilic multicomponent macromolecular brushes is devoted to the study of carbon-chain brushes. Self-organization processes of amphiphilic copolymers in selective solvents cause the formation of a large number of self-organized structures with different morphology. It's spheres, rods, cylinders, tubes, vesicles, disks. ${ }^{\mathbf{1 4 - 1 8}}$ Amphiphilic copolymers are ionic and non-ionic, which is determined by the presence or absence of a charge in a hydrophilic block. In most cases, polyethylene oxide and its derivatives are non-ionic hydrophilic fragments. ${ }^{19-25}$ Long aliphatic or aromatic hydrocarbon radicals can be as a hydrophobic fragment in the structure of an amphiphilic copolymer. There are works on the synthesis of hyperbranched polymers and amphiphilic silica particles. Now, receipt mini-emulsions with side polystyrene branches are the most common works. ${ }^{26-29}$ Amphiphilic silica particles were obtained using tetraethoxysilane by hightemperature reaction gel-sol synthesis in ethylene glycol. ${ }^{30,31}$ Amphiphilic hyperbranched macromolecular compounds may influence polymerization processes ${ }^{32-35}$ and lead to improved homopolymer blends. ${ }^{36-40}$

In the work ${ }^{\mathbf{4 1}}$ to control the interphase properties of glass fibre/PP composites, poly(ethylene glycol) (PEG) chains with different average molar mass were grafted onto the glass fibre surface by treating the fibres with aminopropyltriethoxysilane, 1,6-diisocyanatohexane and PEG stepwise.

Thus, the synthesis, research and use of amphiphilic branched macromolecular substances is the current direction of modern polymer science. The areas of their use are associated with the modification of polymer-forming systems and polymer compositions. Silica particles that contain grafted hydrophobic and hydrophilic branches on the surface are the most effective and universal compounds. Structural homogeneity and regular grafting of amphiphilic side branches can be achieved by using reactive oligomers in reactions with alkoxysilanes.

The aim of the work is the synthesis and characterization of the new amphiphilic branched silica derivatives associated with oligomeric medium. They are of particular interest as modifiers that have a significantly impact influence on the microphase separation processes of block copolymers.

\section{Experimental}

\section{(A) Materials}

Polyoxyethylene glycol (PEO, molecular weight is $400 \mathrm{~g} \mathrm{~mol}^{-1}$ ) was purchased from PJSC Nizhnekamskneftekhim (Nizhnekamsk, Russia), tetraethoxysilane (TEOS) was purchased from CJSC Vekton (St. Petersburg, Russia). Polydimethylsiloxane (PDMS, average molecular weight is $30000 \mathrm{~g} \mathrm{~mol}^{-1}$ ), was purchased from JSC Kazan factory of Synthetic Rubber (Kazan, Russia). Potassium diethylene glycolate (DEG-K) with formula $\mathrm{H}\left(\mathrm{O}-\mathrm{CH}_{2}-\mathrm{CH}_{2}\right)_{2}-\mathrm{OK}$ was obtained in laboratory conditions. The block copolymer of propylene and ethylene oxide (PPEG) with formula $\mathrm{HO}\left[\mathrm{CH}_{2} \mathrm{CH}_{2} \mathrm{O}\right]_{n}\left[\mathrm{CH}_{2}\left(\mathrm{CH}_{3}\right) \mathrm{CH}_{2} \mathrm{O}\right]_{m}\left[\mathrm{CH}_{2} \mathrm{CH}_{2} \mathrm{O}\right]_{n} \mathrm{~K}$, where $n \approx 14$ and $m \approx 48$, molecular weight $4200 \mathrm{~g} \mathrm{~mol}^{-1}$, containing
$30 \mathrm{wt} \%$ of peripheral polyoxyethylene blocks, where the content of potassium alcoholate groups is $10.9 \%$ from the total number of functional groups, was purchased from PJSC Nizhnekamskneftekhim (Nizhnekamsk, Russia). The 2,4-toluene diisocyanate (TDI, 98\%) was purchased from Sigma-Aldrich (St. Louis, MO, USA). PEO, PPEG and PDMS were additionally dried at reduced pressure (approximately $0.1 \mathrm{kPa}$ ) and at elevated temperature of $95{ }^{\circ} \mathrm{C}$ down to $0.01 \%$ moisture concentration. All other reagents were used without further purification.

\section{(B) Synthesis of amphiphilic branched silica derivatives associated with oligomeric medium (ASiP)}

The synthesis of ASiP was multistage. At the first stage, PEO (9.64 ml), TEOS (20 wt\% by weight of the total amount of oligomers and TEOS, $4.76 \mathrm{ml})$, water $(0.04 \mathrm{wt} \%$ of the total weight of components PEG-TEOS, $20 \mu \mathrm{l})$ and DEG-K catalyst $(0.1 \mathrm{wt} \%$ relative to the mass of the total reaction system, $10 \mu \mathrm{l}$ ) were added to the flask. The mixtures were stirred at temperature $90{ }^{\circ} \mathrm{C}$ for at least $6 \mathrm{~h}$. Transetherification of ethoxysilane TEOS's groups, sequential reactions of their hydrolysis with condensation of the resulting $\mathrm{Si}-\mathrm{OH}$ groups simultaneously proceeded in the DEG-K presence and water. Then, the second part of TEOS (10 wt $\%$ by weight of the total amount of TEOS, $2.38 \mathrm{ml}$ ) was introduced into the reaction system. The synthesis was carried out for 6 hours, after the final part of TEOS ( $5 \mathrm{wt} \%$ by weight of the total number of TEOS, $1.4 \mathrm{ml}$ ) was introduced in the mixture with stirring for $4 \mathrm{~h}$. After each stage mixtures were put in vacuum for $10 \mathrm{~min}$ to remove alcohol and water and shift the balance towards the product.

As a result of the described reaction processes, including the interaction of PEO, TEOS with a catalyst DEG-K and water, was obtained silica branched by polyoxyethylene glycol (PEOASiP).

Further, PDMS (5 ml) was added in PEOASiP with stirring at temperature $90{ }^{\circ} \mathrm{C}$, after which the viscosity increased in reaction system and ASiP was obtained.

\section{(C) Synthesis of modified by ASiP microporous polymers based on TDI and PPEG}

The reaction was carried out in toluene at $20{ }^{\circ} \mathrm{C}$ in a flask equipped with a reflux condenser. The polymerization proceeded with the constant stirring, a magnetic stirrer being used. PPEG (1 g), toluene (7.9 g), bisphenol A (0.004 g), acetic acid $(0.007 \mathrm{~g})$ and calculated amount of ASiP were added to the flask. The reaction mass was mixed at a given temperature until the complete dissolution of PPEG took place, then TDI ( $0.62 \mathrm{~g}$.) at a molar ratio [PPEG]: [TDI] $=1: 15$ was introduced. Five minutes after mixing with TDI, triethylamine $(0.0072 \mathrm{~g})$ and water $(0.001 \mathrm{~g})$ were loaded into the reaction system. After ten minutes the reaction mass was dispensed to Petri dish, and then it was cured at room temperature during 72 hours.

\section{(D) Polymer characterization}

Fourier transform infrared (FTIR) spectroscopy analysis. The FTIR spectra were recorded on an InfraLUM FT 08 Fourier transform spectrometer (Lumex, St. Petersburg, Russia) using the attenuated total reflection technique in the spectral range of 
$3800-600 \mathrm{~cm}^{-1}$. The spectral resolution was $2 \mathrm{~cm}^{-1}$ and the number of scans was 16 .

Thermal gravimetric analysis (TGA). TGA was performed using STA-600 TGA-DTA combined thermal analyzer (PerkinElmer, Waltham, MA, USA). The samples $(0.1 \mathrm{~g})$ were loaded in alumina pans and heated from 30 to $750{ }^{\circ} \mathrm{C}$ at a rate of 5 $\mathrm{K} \mathrm{min}^{-1}$ in a nitrogen atmosphere.

Dynamic light scattering (DLS). Dynamic light scattering experiments were carried out on Zetasizer Nano ZS (Malvern, Great Britain). This instrument has $4 \mathrm{mV} \mathrm{He}-\mathrm{Ne}$ laser, which is working on $632.8 \mathrm{~nm}$ wavelength. Measurements were carried out at $173^{\circ}$ detection angle. The experiments were carried out at $25{ }^{\circ} \mathrm{C}$ in the disposable plastic cuvettes of $1 \mathrm{~cm}$ path length.

NMR spectroscopy. The ${ }^{1} \mathrm{H}$ and ${ }^{29} \mathrm{Si}$ NMR spectra were registered on a Bruker Avance III $400 \mathrm{MHz}$ (Bruker, Germany) NMR spectrometer at $400.1 \mathrm{MHz}$ for ${ }^{1} \mathrm{H}$ and $79.5 \mathrm{MHz}$ for ${ }^{29} \mathrm{Si}$ (chemical shifts are relative to $\mathrm{Me}_{4} \mathrm{Si}$ in both cases). Solvent is $\mathrm{CDCl}_{3}$. Chemical shifts are indicated in ppm.

SEM analyses. Analytical Autoemission scanning electron microscope Merlin (Carl Zeiss, Germany) was employed to examine the surfaces morphology of samples. The electron microscope is based on the core of the new generation of GEMINI II, which generates an electron beam. The working energy of the primary electron radiation was $5 \mathrm{kV}$, the working distance was $6.1 \mathrm{~mm}$. Spatial resolution is $200 \mathrm{~nm}$. The range of operating currents is $300 \mathrm{pA}$. Magnification range is $50.00 \mathrm{k} \times$ (in SE mode). Built-in detector is In-lens SE. Platinum was sprayed onto the surface of the samples.

Tensile stress - strain measurements. Tensile stress - strain measurements were obtained from the film samples with dimensions of $40 \mathrm{~mm} 15 \mathrm{~mm}$ using Universal Testing Machine Inspekt mini (Hegewald \& Peschke Meß- und Prüftechnik $\mathrm{GmbH}$, Nossen, Germany) at $293 \pm 2 \mathrm{~K}$ and $1 \mathrm{kN}$. The crosshead speed was set at $50 \mathrm{~mm} \mathrm{~min}{ }^{-1}$ and the test continued until sample failure. Minimums of five tests were analyzed for each sample and the average values were reported.

Mechanical loss tangent measurements (MLT). The MLT curves of polymer samples were taken using the dynamic mechanical analyzer Netzsch DMA 242 (Germany) in the mode of oscillating load. Force and stress-stain correspondence were calibrated using a standard mass. The thickness of the sample was $2 \mathrm{~mm}$. Viscoelastic properties were measured under nitrogen. The samples were heated from $-100{ }^{\circ} \mathrm{C}$ to $300{ }^{\circ} \mathrm{C}$ at the rate of $3 \mathrm{~K} \mathrm{~min}^{-1}$ and frequency of $1 \mathrm{~Hz}$. The mechanical loss tangent was defined as the ratio of the viscosity modulus $G^{\prime \prime}$ to the elasticity modulus $G^{\prime}$.

Thermomechanical analysis (TMA). TMA curves of polymer samples were obtained using TMA 402 F (Netzsch, Germany) thermomechanical analyzer in the compression mode. The thickness of the sample was $2 \mathrm{~mm}$, the rate of heating was 3 $\mathrm{K} \min ^{-1}$ from $-100{ }^{\circ} \mathrm{C}$ to $300{ }^{\circ} \mathrm{C}$ in static mode, and the load was $2 \mathrm{~N}$.

Density measurement. To determine the density of the films was used automatic densitometer H-200L (TaiwanGermany). It has a resolution $\left(0.0001 \mathrm{~g} \mathrm{~cm}^{-3}\right)$ based on Sartorius sensitive analytical scales. Measurement time is $20 \mathrm{~s}$. The measurement principle is hydrostatic weighing.
Specific gravity is defined as the weight ratio of material volume to the weight of an equivalent water volume at $20^{\circ} \mathrm{C}$.

Measurement of critical micelle concentration (CMC). The droplet counting method was used to determine the surface tension $(\sigma)$. The basis of the calculations is the law, where the weight of the drop that comes off the pipette is proportional to the surface tension of the fluid and the radius of the pipette $(R)$ : $m=2 \pi R \sigma / g$, where: $g$ is acceleration of gravity; $m$ is the drop mass of the test liquid.

\section{Results and discussion}

\section{(A) ASiP characterization}

The reagents were preliminarily examined to analyze the size distribution of ASiP particles. PEO in toluene exists in the associated state. This is the reason that the average size of PEO determined using dynamic light scattering was $450 \mathrm{~nm}$ (Fig. 1a). The average particle size of the PDMS is $10 \mathrm{~nm}$ (Fig. 1b). The average particle size of PEOASiP is $60-400 \mathrm{~nm}$ with a maximum at $150 \mathrm{~nm}$. There are particles with a size distribution in the region of 10-30 $\mathrm{nm}$, which exhibit relatively low intensity (Fig. 1c).

In the case of $\mathrm{ASiP}$, there is an increase in the proportion of particles with a size distribution in the region of $10-30 \mathrm{~nm}$. The intensity in the region of the size distribution of $60-400 \mathrm{~nm}$ falls from 8 to $5.5 \%$ compared with PEOASiP (Fig. 1d). There is no increase in particle size. This is because of PEOASiP is associated in toluene due to intermolecular interactions of PEO. As a result, the share of associates falls because of PDMS addition to PESOASiP and consequently ASiP formation.

From these results we expect that PEOASiP particles exist in the associated state. The sizes of associates are $60-400 \mathrm{~nm}$. The redistribution of intensity (a decrease in the region of 60$400 \mathrm{~nm}$ and an increase in the region of $10-30 \mathrm{~nm}$ ) is associated with the consumption of PEOASiP particles for the formation of ASiP particles.

${ }^{29} \mathrm{Si}$ NMR and ${ }^{1} \mathrm{H}$ NMR spectra were measured for TEOS, PDMS and ASiP. The ${ }^{1} \mathrm{H}$ NMR spectra of the TEOS (Fig. 2) contains triplets at $\delta=1.224 \mathrm{ppm}$ and $\delta=3.84 \mathrm{ppm}$ assigned to the protons of the methyl and methylene groups, respectively. The ${ }^{1} \mathrm{H}$ NMR spectra of PDMS (Fig. 3) exhibits a resonance signal at $\delta=0.066 \mathrm{ppm}$, corresponding to the protons of the methyl groups in the $\mathrm{CH}_{3}-\mathrm{Si}-\mathrm{CH}_{3}$. The ${ }^{1} \mathrm{H}$ NMR spectrum of ASiP (Fig. 4), the signal at $\delta=1.224 \mathrm{ppm}$ almost disappears, corresponding to the protons of the methyl groups of TEOS. There is a signal at $\delta=3.7-3.5 \mathrm{ppm}$ assigned to the protons of methylene groups in the composition of PEO links. According to the ${ }^{1} \mathrm{H}$ NMR spectra, low intensity peaks are present in the ASiP composition and corresponds to the presence of unreacted TEOS and the absence of ethanol (the product of TEOS hydrolysis). At the ${ }^{29} \mathrm{Si}$ NMR spectra of the TEOS, a signal is present at $\delta=-81.963$ ppm (Fig. 5).

Analysis of the ${ }^{29} \mathrm{Si}$ NMR spectra of PEOASiP confirms the absence of TEOS in the ASiP (Fig. 6). The absence of signals at $\delta=-82 \mathrm{ppm}$ enables to conclude that tetraalkoxide silane derivatives are practically absent in the composition of ASiP. 


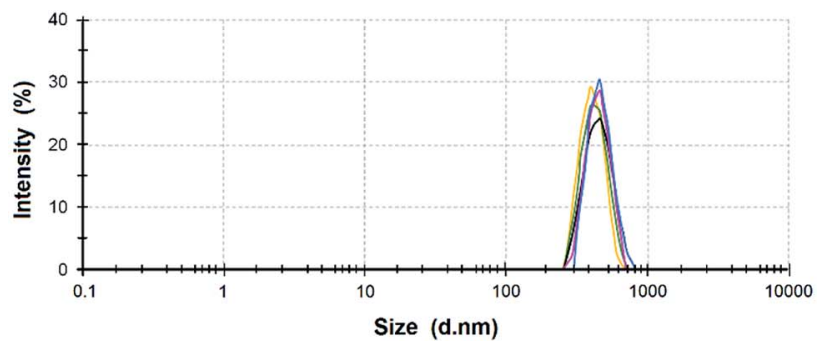

(a)

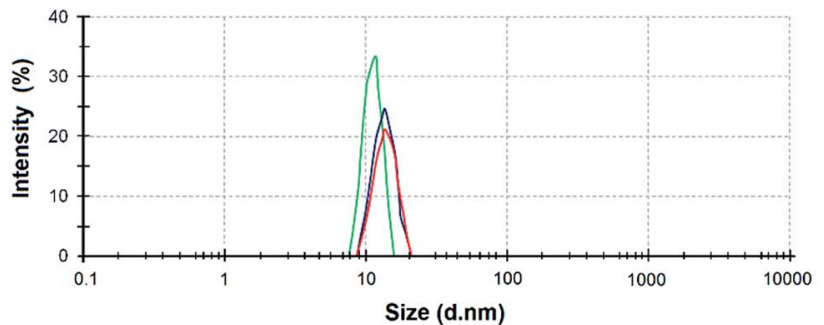

(b)

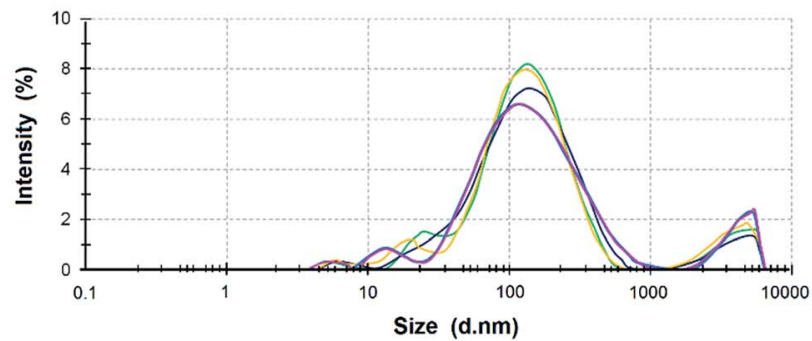

(c)

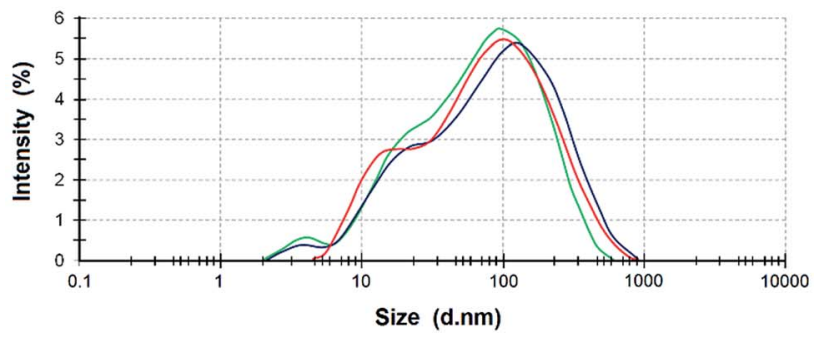

(d)

Fig. 1 Particle size distribution of PEO (a), PDMS (b), PEOASiP (c), ASiP (d) in toluene.

The appearance of the $\delta=-108 \mathrm{ppm}$ signal indicates the formation of the cube silica, substituted by alkoxide groups. ${ }^{42}$

The reactions occurring during the formation of PEOASiP and ASiP are shown at Scheme 1.

The bands at 965,1075 , and $1102.5 \mathrm{~cm}^{-1}$ are present on the TEOS FTIR spectra, the corresponding valence vibrations of the $\mathrm{Si}-$ $\mathrm{O}$ bonds in the $\mathrm{Si}-\mathrm{O}-\mathrm{C}$ group of tetraethoxysilan (Fig. 7). Absorption band at $1010 \mathrm{~cm}^{-1}$ corresponding to the stretching vibrations of the $\mathrm{Si}-\mathrm{C}$ bond (as part of the $\mathrm{Si}-\mathrm{CH}_{3}$ group) and band at $1070 \mathrm{~cm}^{-1}$ assigned to the stretching vibrations of the $\mathrm{Si}-\mathrm{O}$ bond in the composition of the $\mathrm{Si}-\mathrm{O}-\mathrm{Si}$ present in the FTIR spectra of PDMS.

On the FTIR spectra of ASiP, the band at $965 \mathrm{~cm}^{-1}$ corresponding to the stretching vibrations of the $\mathrm{Si}-\mathrm{O}$ bond in the $\mathrm{Si}-\mathrm{O}-$ $\mathrm{C}$ group of TEOS has completely changed shape and location. The

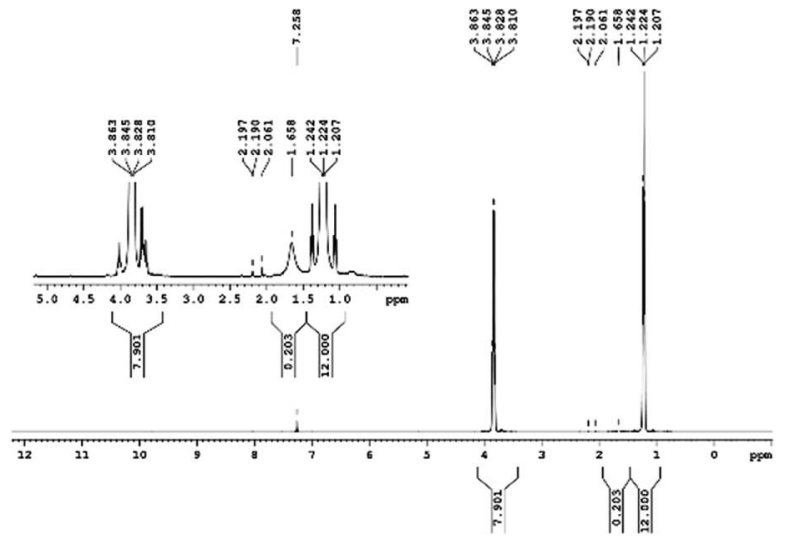

Fig. $2{ }^{1} \mathrm{H}$ NMR spectra of TEOS.

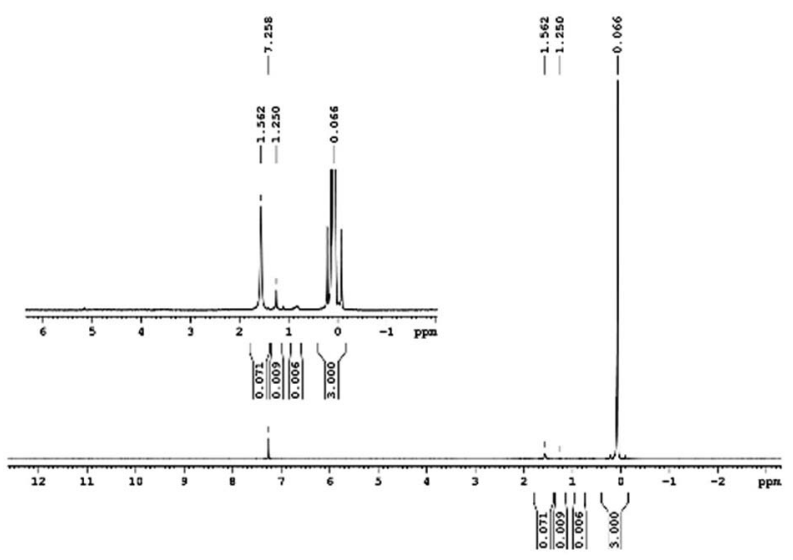

Fig. $3{ }^{1} \mathrm{H}$ NMR spectra of PDMS

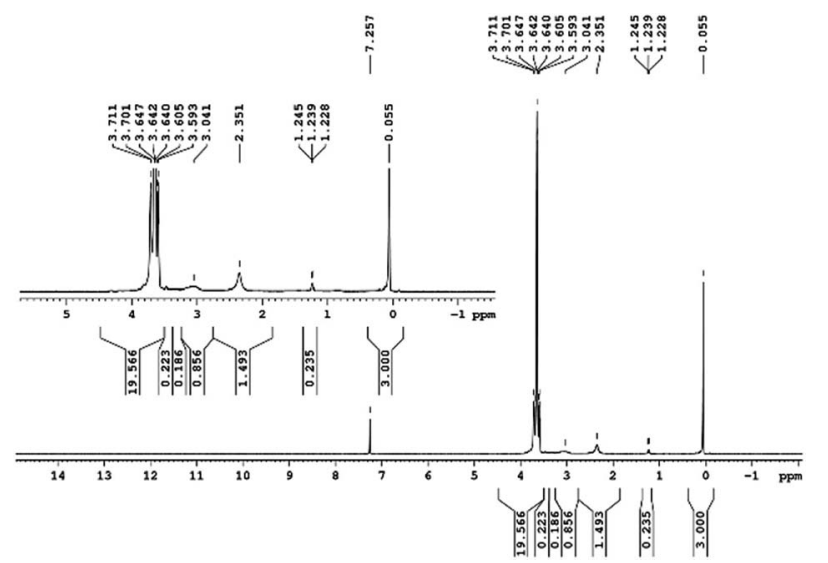

Fig. $4{ }^{1} \mathrm{H}$ NMR spectra of ASiP.

spectral manifestation turned out to be more gentle and it's the superposition of the absorption bands at 950 and $965 \mathrm{~cm}^{-1}$. It can be due to the existence of the $\mathrm{Si}-\mathrm{O}-\mathrm{C}$ group, but not as part of TEOS. The bands at 1075 and $1100 \mathrm{~cm}^{-1}$ correspond to the stretching vibrations of the $\mathrm{Si}-\mathrm{O}$ bond in the $\mathrm{Si}-\mathrm{O}-\mathrm{C}$ group. It indicates the existence of the group $\mathrm{Si}-\mathrm{O}-\mathrm{C}$ in the composition of polyoxyethylene 


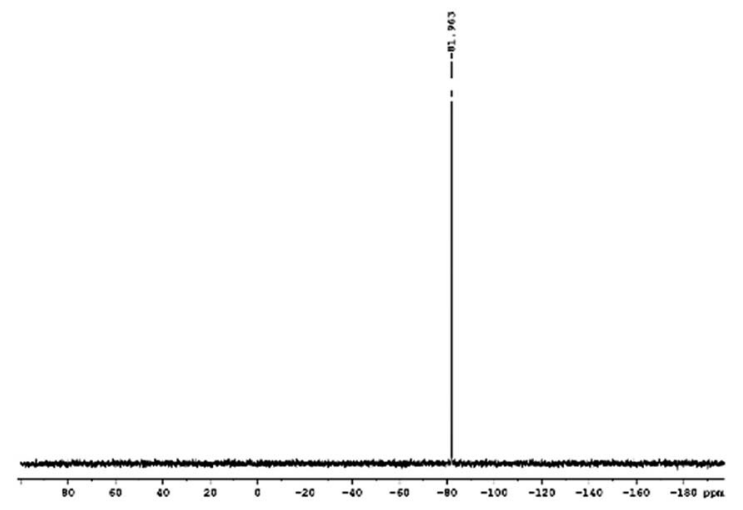

Fig. $5{ }^{29}$ Si NMR spectra of TEOS.
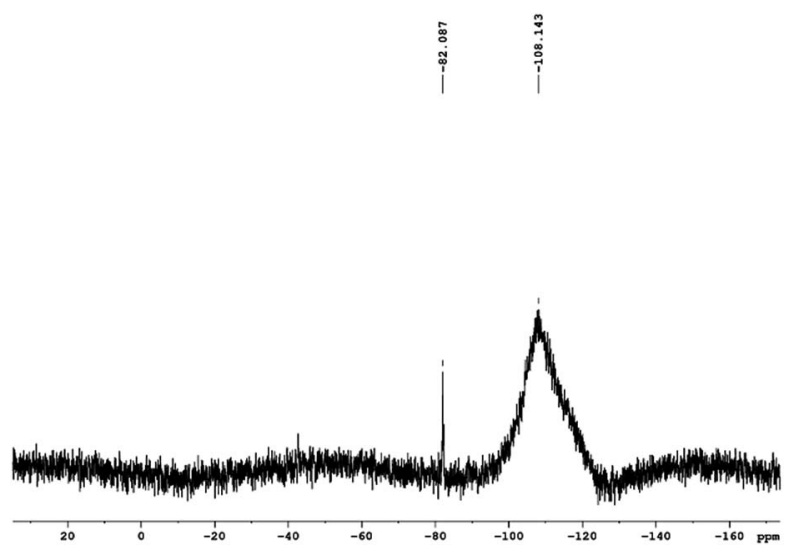

Fig. $6{ }^{29}$ Si NMR spectra of PEOASiP.
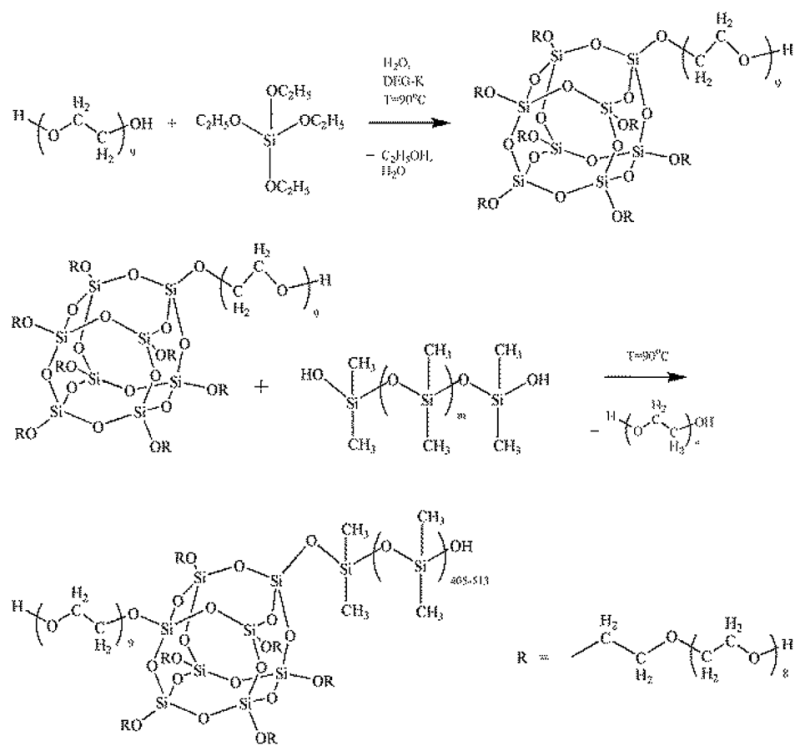

Scheme 1 The scheme of experimental approaches to form ASiP.

silane fragments ASiP. It should be noted that the intensities of these bands differ slightly compared with TEOS, and the band at $1102.5 \mathrm{~cm}^{-1}$ has shifted to $1100 \mathrm{~cm}^{-1}$. PEO and PDMS are attached

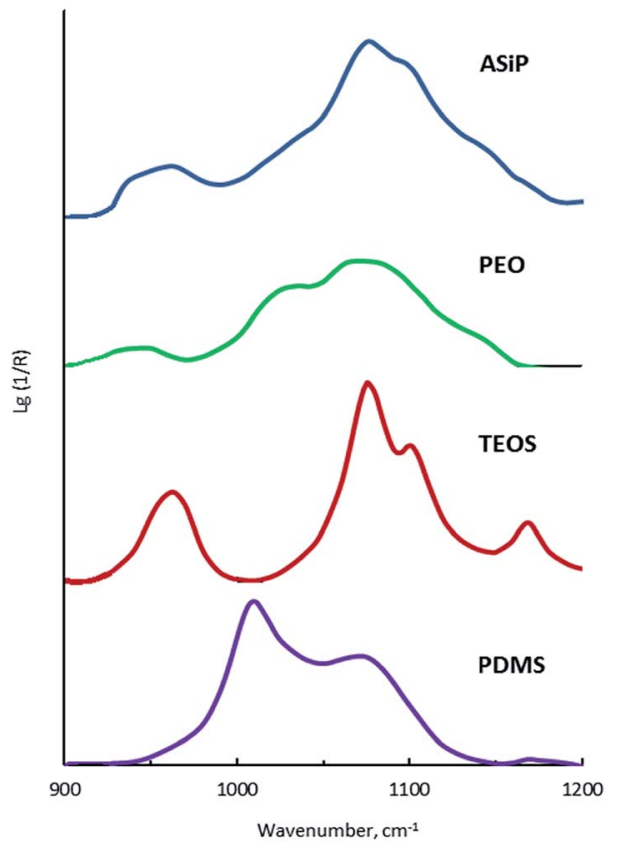

Fig. 7 FTIR spectra of TEOS, PEO, PDMS and ASiP.

to a cubic silica derivative. It can be judged by the characteristic absorption regions for the $\mathrm{Si}-\mathrm{O}-\mathrm{Si}$ bonds in cubic structures ${ }^{42}$ that are present in the FTIR spectra of ASiP. These are pronounced shoulders in the region of $1140,1179 \mathrm{~cm}^{-1}$ and a low-intensity band at $1200 \mathrm{~cm}^{-1}$.

\section{(B) Thermal - gravimetric behavior of PEOASiP and ASiP}

The beginning of the mass loss for PEOASiP and ASiP is in the region of $150{ }^{\circ} \mathrm{C}$ (Fig. 8). The maximum decomposition rate of PEOASiP starts at $390{ }^{\circ} \mathrm{C}$ and for ASiP is observed at a temperature that exceeds the maximum decomposition rate of PEOASiP by $20^{\circ} \mathrm{C}$. PEOASiP shows a lower heat resistance compared to ASiP due to the fact that in this case cubic silica has only polyoxyethylene environment. Partial substitution of PEO with PDMS naturally leads to an increase in the heat resistance of ASiP.

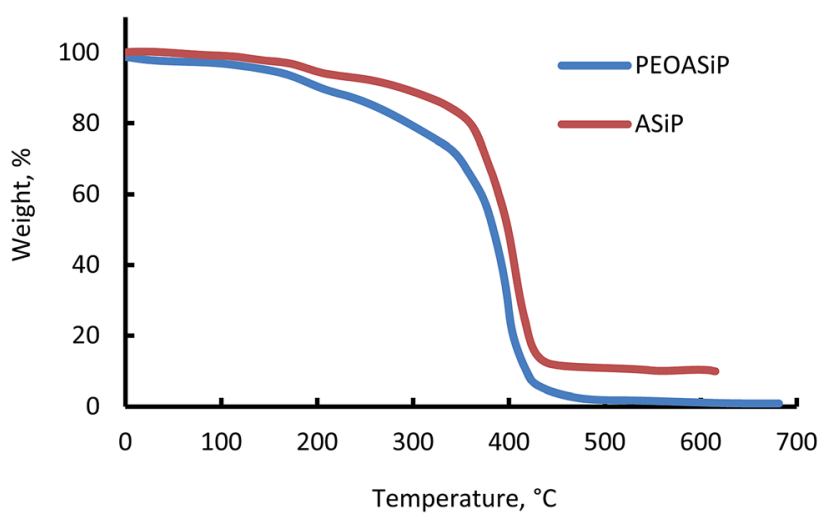

Fig. 8 TGA of PEOASiP and ASiP. 


\section{(C) Surface active properties}

An important feature of amphiphilic compounds is the ability to self-organize processes, which are accompanied by the formation of micelles. The self-assembly of small-molecule amphiphiles has been studied for many decades, and various morphologies have been observed in bulk and in aqueous solutions. The morphologies include spherical and cylindrical micelles, lamellae and vesicles, among others. ${ }^{43-45}$

Amphiphilic polymers form thermodynamically more stable structures in comparison with low-molecular surfactants. Values of $\mathrm{CMC}$ of polymer micelles are, as a rule, $10^{-6}$ to $10^{-7} \mathrm{M}$, while for low molecular weight surfactants this value is of the order $10^{-3}$ to $10^{-4} \mathrm{M}{ }^{46}$ Structures of amphiphilic block copolymers are also kinetically more stable, since their dissociation into individual macromolecules is slowed down. ${ }^{47}$

According to measurements of surface tension, ASiP exhibit the ability to form micelles in water (Fig. 9). All particles are characterized the manifestation of two CMC because of their ability to form different micellar structure with a change of ASiP concentration.

It turned out that the content of PDMS in the ASiP composition influences the values of CMC and surface tension. Values of CMC and surface tension fall to a certain value with increasing of PDMS content in the composition of ASiP. The critical micelle concentration for ASiP, obtained using $0 \%, 1 \%$, $6 \%$ and $21.6 \%$ of PDMS are $1010^{-4}, 9910^{-5}, 3310^{-5}$ and 2.5 an $10^{-5} \mathrm{M}$, respectively.

This fact confirms that PDMS is included in ASiP and enhances their amphiphilicity. The scheme of ASiP formation and the scheme of ASiP micelle formation in water are shown in Schemes 2 and 3, respectively.

\section{(D) SEM analyses}

The ASiP aqueous emulsion was treated with ultrasound for SEM analysis, and then a drop of the emulsion was placed on the substrate. The measurements were carried out after preliminary evacuation of the sample (Fig. 10).

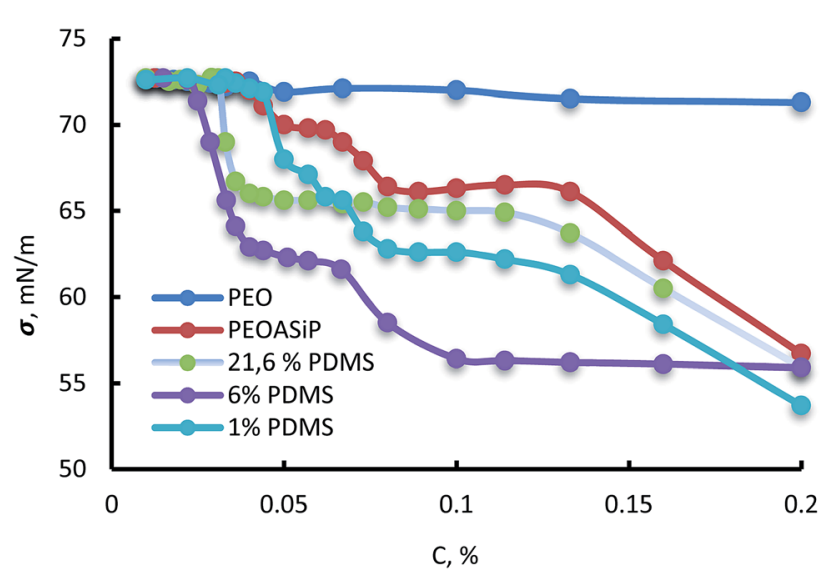

Fig. 9 Dependencies of surface tension $\left(\sigma, \mathrm{mN} \mathrm{m}^{-1}\right)$ on concentration in water $(C, \%)$ for PEO, PEOASiP and ASiP, obtained using $1 \%$ PDMS, $6 \%$ PDMS, $21.6 \%$ PDMS.

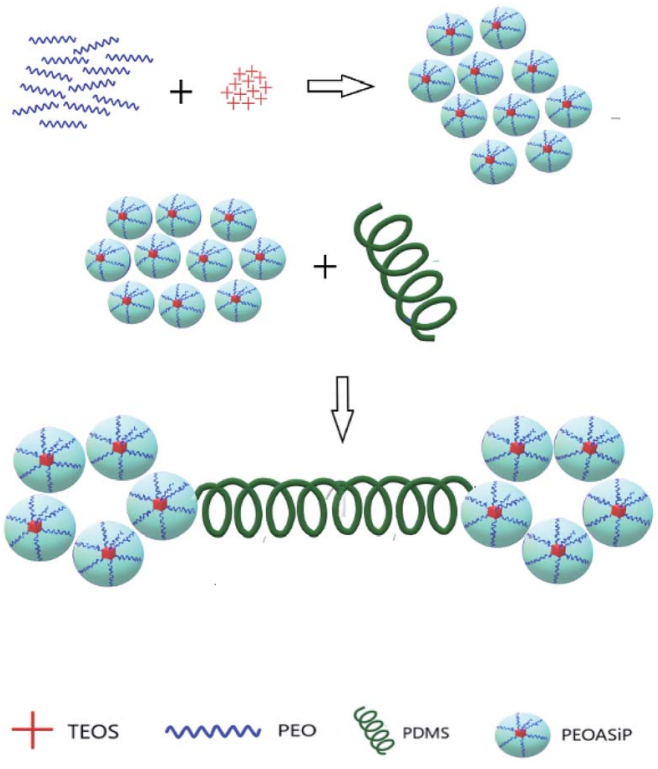

Scheme 2 The scheme of ASiP formation.

The particle diameter is $20-70 \mathrm{~nm}$. According to the SEM measurements, the particles of the prepared aqueous emulsion are uniform in size; the internal component of the particles (core) is denser than the shell. The size of the prepared aqueous emulsion determined using SEM correlates with the size of the ASiP in toluene determined using dynamic light scattering method. The particle size distribution of ASiP determined using DLS measurements are in the range of 10-30 nm (Fig. 1d).

\section{(E) Modification of polymers based on TDI and PPEG with ASiP}

ASiP was used to modify microporous polymers based on TDI and PPEG. These polymers are of interest due to their high gas transport characteristics. ${ }^{48-54}$ The specific characteristics of theirs supramolecular structure is polyisocyanate blocks of acetal nature (O-polyisocyanates) as a component with a rigid chain. A scheme of polymers synthesis by the reaction of

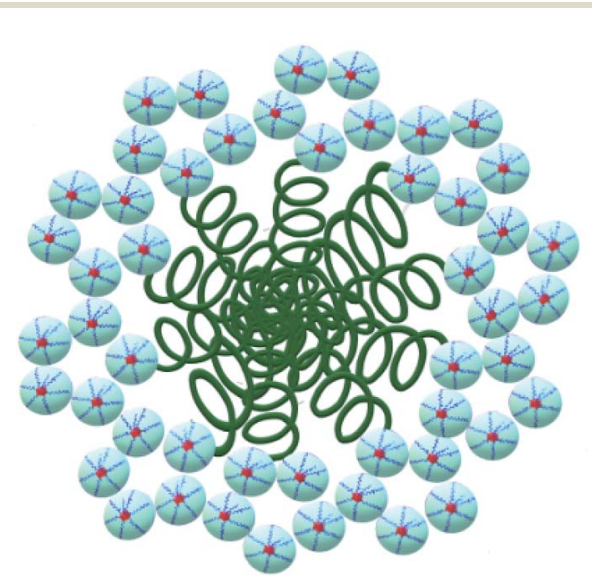

Scheme 3 The structure of ASiP micelles. 


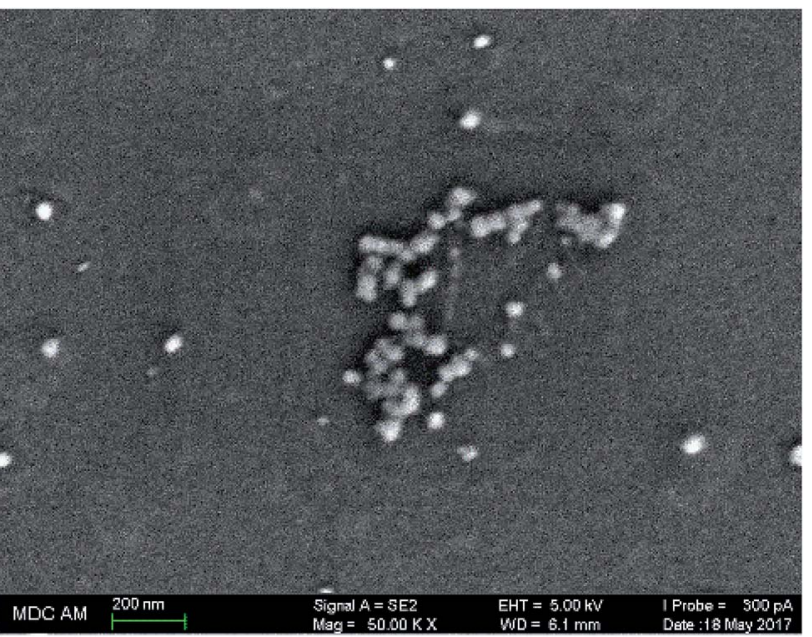

Fig. 10 SEM image of ASiP aqueous emulsion.

polyaddition of 2,4-toluene diisocyanate (TDI) and a macroinitiator, which is a potassium-substituted block copolymer of propylene and ethylene (PPEG) is shown in Scheme 4.

Microporous polymers modified with ASiP was investigated using FTIR spectroscopy. There aren't $\mathrm{C}=\mathrm{O}$ bonds in the structure of $O$-polyisocyanates. Thus, the $\mathrm{N}=\mathrm{C}$ bond, which exists in the structure, is characterized by a band at $1670 \mathrm{~cm}^{-1}$. According to Fig. 11, changes in the area of $1670 \mathrm{~cm}^{-1}$ aren't observed and the intensity of the band in the area of $1710 \mathrm{~cm}^{-1}$ doesn't increase. It's characteristic of polyisocyanurates, which are formed during a possible competing reaction. It leads to the disclosure of the $\mathrm{N}=\mathrm{C}=\mathrm{O}$ group by the $\mathrm{N}=\mathrm{C}$ component and

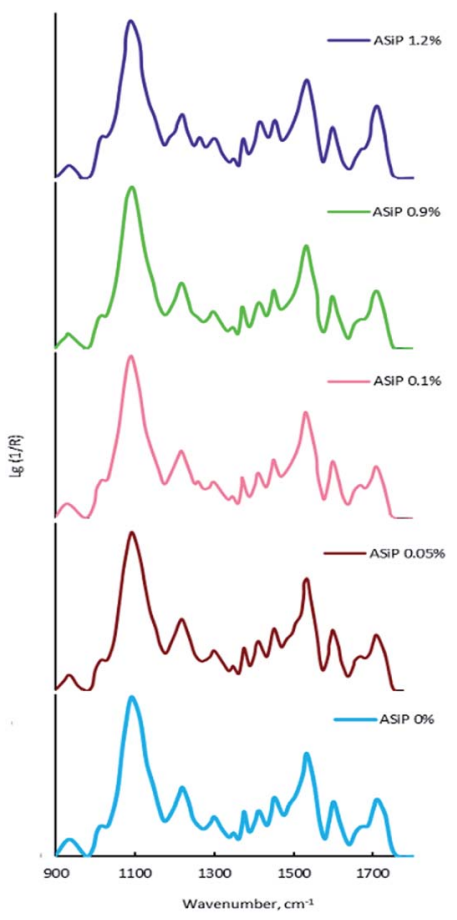

Fig. 11 FTIR spectra of polymers.

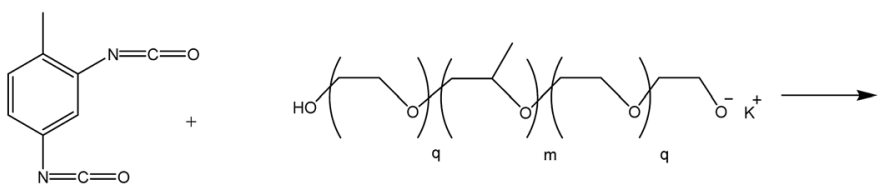
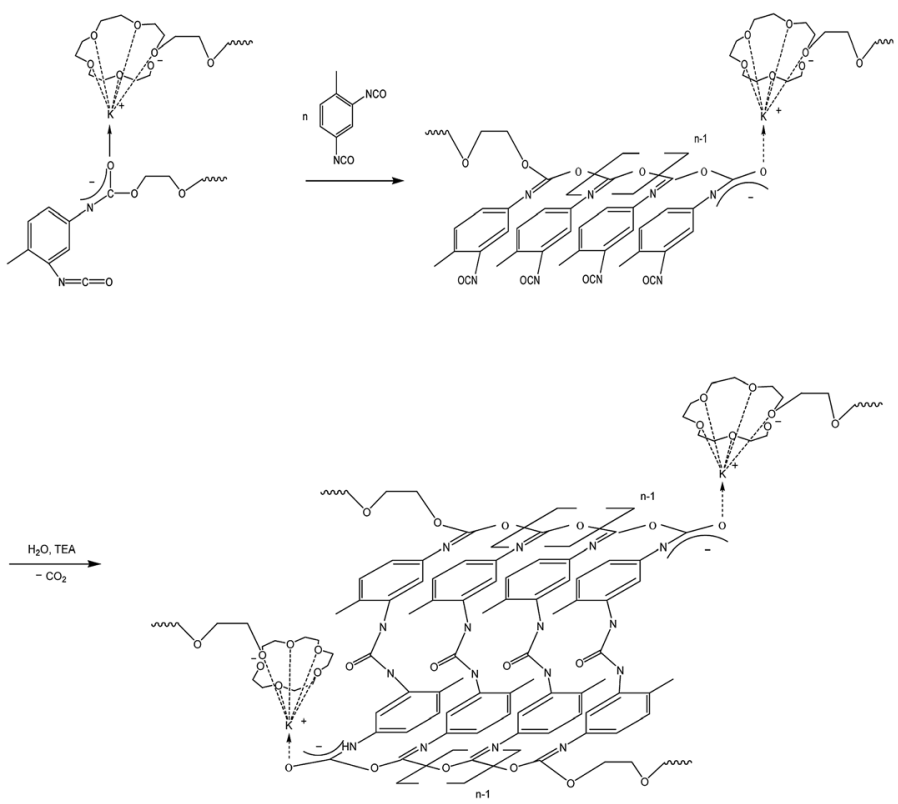

Scheme 4 Synthesis of polymers based on TDI and PPEG. 


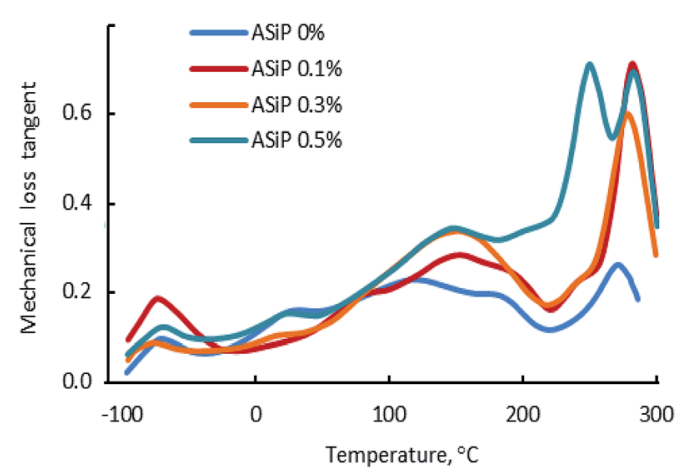

(a)

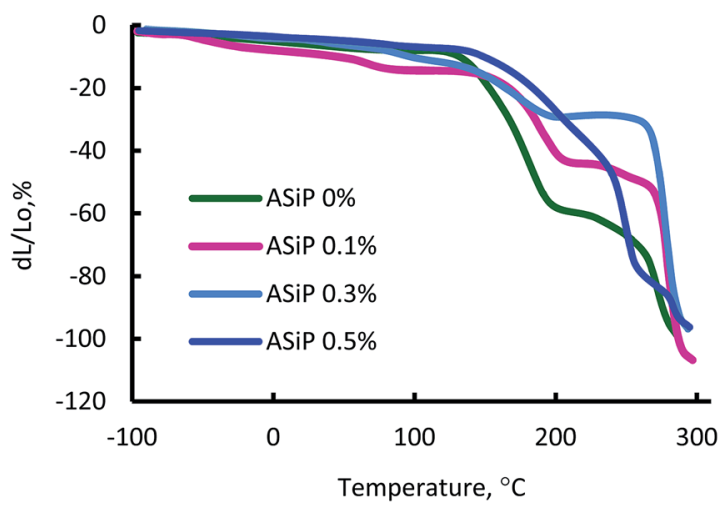

(b)

Fig. 12 MLT (a) and TMA (b) curves of polymers.

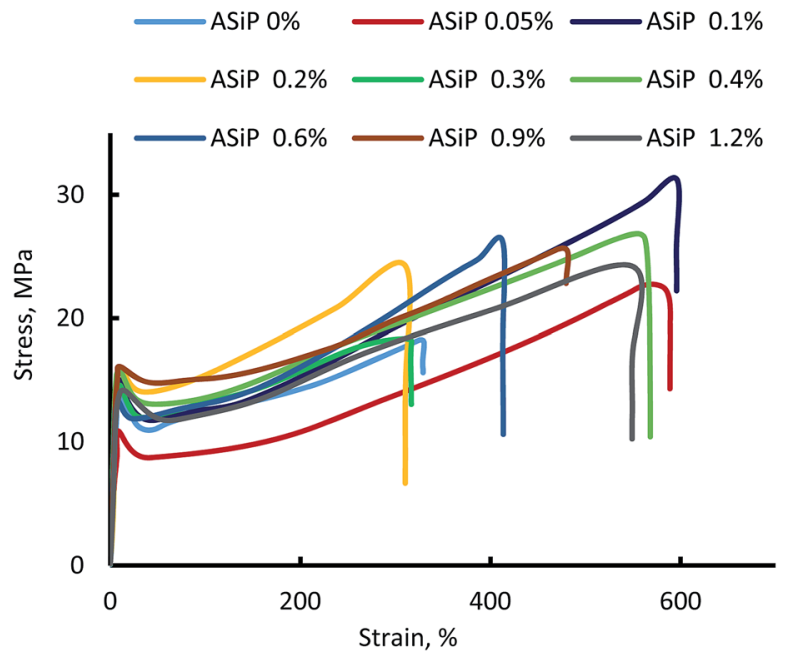

Fig. 13 Tensile tests of polymers.

the use of ASiP doesn't interfere with the reaction by the mechanism, which is presented in Scheme 4.

\section{(F) Thermomechanical and dynamic mechanical analysis of polymers}

According to Fig. 12a, the use of ASiP has a significant impact on the thermomechanical behaviour of polymers.
Table 1 Density and Young's modulus $(E)$ of polymers

\begin{tabular}{lll}
\hline$[\mathrm{ASiP}], \mathrm{wt} \%$ & Density, $\mathrm{g} \mathrm{cm}^{-3}$ & $E, \mathrm{MPa}$ \\
\hline 0.00 & 1.156 & 198 \\
0.03 & 1.112 & 198 \\
0.05 & 1.096 & 217 \\
0.08 & 1.129 & 349 \\
0.10 & 1.165 & 469 \\
0.20 & 1.167 & 121 \\
0.30 & 1.168 & 413 \\
0.40 & 1.163 & 219 \\
0.60 & 1.158 & 313
\end{tabular}

This figure depicts, that the region of the $\alpha$-transition, which corresponds to the beginning of the segmental mobility of the flexible polyether component, practically unchanged, but significant changes are observed in the region of relatively high temperatures. This is an increase of the structural elements mobility of the polymer matrix in the region of $60-150{ }^{\circ} \mathrm{C}$. The most significant deformation effect is observed in the temperature range above $200{ }^{\circ} \mathrm{C}$, which corresponds to relaxation processes with the participation of polyisocyanate hard blocks. Modification of films by ASiP to its content of $0.3 \mathrm{wt} \%$ leads to a decrease in the strain from $60 \%$ to $20 \%$ (Fig. 12b). Such a significant effect occurs because of the enhancement of intermolecular interactions and the overall structural organization of the rigid-chain component, which are $O$ polyisocyanates.

\section{(G) Mechanical behavior and density of polymers}

Modification of polymers based on TDI and PPEG by ASiP leads to an increase in strength, relative elongation (Fig. 13), and decrease in density in the low concentrations of ASiP (Table 1). The decrease in the density of microporous polymers is a consequence of the active influence of ASiP on the processes of their supramolecular organization.

\section{Conclusions}

Amphiphilic branched silica derivatives associated with oligomeric medium were obtained using tetraethoxysilane, polyoxyethylene and low molecular weight polydimethylsiloxane. The creation of a silica core was based on TEOS hydrolysis and condensation reactions using latent water and a DEG-K catalyst. These reactions proceeded with the sequential participation of PEO and PDMS in parallel transetherification reactions.

Microporous polymers based on 2,4-toluene diisocyanate and block copolymers of propylene and ethylene oxides terminated by potassium-alcoholate groups were modified by ASiP. It has been shown that ASiP at the phase interface between thermodynamically incompatible macrochains performs the function of a link. It leads to a significant increase of intermolecular interactions, the structural organization of the rigid-chain component and the growth of the mechanical properties of the modified microporous polymers. The decrease in the density of microporous polymers is a consequence of the active 
influence of ASiP on the processes of their supramolecular organization.

\section{Abbreviations}

$\begin{array}{ll}\text { PEO } & \text { Polyoxyethylene glycol } \\ \text { TEOS } & \text { Tetraethoxysilane } \\ \text { DEG-K } & \text { Potassium diethylene glycolate } \\ \text { PDMS } & \text { Polydimethylsiloxane } \\ \text { PEOASiP } & \text { Silica branched by polyoxyethylene glycol } \\ \text { ASiP } & \text { Amphiphilic branched silica derivatives associated } \\ & \text { with oligomeric medium } \\ \text { TDI } & \text { 2,4-Toluene diisocyanate } \\ \text { PPEG } & \text { Potassium-substituted block copolymer of propylene } \\ & \text { and ethylene oxide } \\ \text { FTIR } & \text { Fourier transform infrared spectroscopy analysis } \\ \text { TGA } & \text { Thermogravimetric analysis } \\ \text { NMR } & \text { Nuclear magnetic resonance } \\ \text { SEM } & \text { Scanning electron microscopy } \\ \text { TMA } & \text { Thermomechanical analysis } \\ \text { MLT } & \text { Mechanical loss tangent } \\ \text { CMC } & \text { Critical micelle concentration }\end{array}$

\section{Conflicts of interest}

There are no conflicts to declare.

\section{Acknowledgements}

The reported study was funded by RFBR according to the research project no. 18-43-160002.

\section{References}

1 W. Zhao, P. Fonsny, P. Fitzgerald, G. G. Warr and S. Perrier, Polym. Chem., 2013, 4, 2140.

2 J. Wang, K. Yao, C. Wang, C. Tang and X. Jiang, J. Mater. Chem. B, 2013, 1, 2324.

3 S. Sheiko, B. S. Sumerlin and K. Matyjaszewski, Prog. Polym. Sci., 2008, 33, 759.

4 S. S. Sheiko, S. A. Prokhorova, K. L. Beers, K. Matyjaszewski, I. I. Potemkin, A. R. Khokhlov and M. Möller, Macromolecules, 2001, 34(23), 8354.

5 A. V. Yakimansky, T. K. Meleshko, D. M. Ilgach, M. A. Bauman, T. D. Anan'eva, L. G. Klapshina, S. A. Lermontova, I. V. Balalaeva and W. E. Douglas, $J$. Polym. Sci., Part A: Polym. Chem., 2013, 51, 4267.

6 A. Krasova, E. Belyaeva, E. Tarabukina, A. Filippov, T. Meleshko, D. Ilgach, N. Bogorad and A. Yakimansky, Macromol. Symp., 2012, 316(1), 32.

7 M. Zhang and A. H. E. Müller, J. Polym. Sci., Part A: Polym. Chem., 2005, 43, 3461.

8 H. Gao and K. Matyjaszewski, J. Am. Chem. Soc., 2007, 129(20), 6633.

9 M. Schappacher and A. Deffieux, Science, 2008, 319(5869), 1512.
10 Y. Yan, Y. Shi, W. Zhu and Y. Chen, Polymer, 2013, 54(21), 5634.

11 K. Matyjaszewski and N. V. Tsarevsky, J. Am. Chem. Soc., 2014, 136(18), 6513.

12 H.-Y. Chang, Y.-L. Lin and Y.-J. Sheng, Macromolecules, 2012, 45(11), 4778.

13 X. Lian, D. Wu, X. Song and H. Zhao, Macromolecules, 2010, 43(18), 7434.

14 J. Raez, I. Manners and M. A. Winnik, J. Am. Chem. Soc., 2002, 124, 10381.

15 Y. Mai and A. Eisenberg, Chem. Soc. Rev., 2012, 41, 5969.

16 S. J. Holder and N. A. J. M. Sommerdijk, Polym. Chem., 2011, 2, 1018.

17 M. Zhang and A. H. E. Müller, J. Polym. Sci., Part A: Polym. Chem., 2005, 43(16), 3461.

18 A. P. Martinez, Zh. Cui, Ch. Hire, T. A. P. Seery and D. H. Adamson, Macromolecules, 2015, 48(13), 4250.

19 M. Higa, K. Yaguchi and R. Kitani, Electrochim. Acta, 2010, 55(4), 1380.

20 A. H. E. Müller, Y. Cai, M. Hartenstein, M. Gradzielski, M. Zhang, H. Mori and D. V. Pergushov, Polym. Prepr., 2004, 45(2), 267.

21 D. Neugebauer, Polymer, 2007, 48(17), 4966.

22 G. Wu, S. C. Chen, Q. Zhan and Y. Z. Wang, Macromolecules, 2011, 44(4), 999.

23 F. P. Wang, X. Z. Du, H. P. Mu, D. X. Zhang and Y. J. Ma, Adv. Mater. Res., 2011, 233-235, 2138.

24 Zh. Cheng, X. Zhu, E. T. Kang and K. G. Neoh, Langmuir, 2005, 21(16), 7180.

25 J. Zhao, G. Mountrichas, G. Zhang and S. Pispas, Macromolecules, 2010, 43(4), 1771.

26 W. Lu, M. Chen and L. M. Wu, J. Colloid Interface Sci., 2008, 328, 98.

27 J. N. Zhang, M. Y. Wu, Q. Y. Wu, J. J. Yang, N. N. Liu and Z. L. Jin, Chem. Lett., 2010, 39(3), 206.

28 C. L. Zhang, B. Liu, C. Tang, J. G. Liu, X. Z. Qu, J. L. Li and Z. Z. Yang, Chem. Commun., 2010, 46(1), 4610.

29 M. Teo, S. K. Suh, T. A. Hatton, Ma. Ashokkumar and F. Grieser, Langmuir, 2011, 27(1), 30.

30 L. Zhang, F. Zhang, W. F. Dong, J. F. Song, Q. S. Huob and H. B. Sun, Chem. Commun., 2011, 47(4), 1225.

31 M. M. Rahman, F. Montagne, H. Fessi and A. Elaissari, Soft Matter, 2011, 7, 1483.

32 N. B. Bowden, M. Dankova, W. Wiyatno, C. J. Hawker and R. M. Waymouth, Macromolecules, 2002, 35(25), 9246.

33 A. Walther, M. Hoffmann and A. H. E. Muller, Angew. Chem., Int. Ed., 2008, 47(4), 711.

34 J. Y. Yuan, Y. Y. Xu, A. Walther, S. Bolisetty, M. Schumacher, H. Schmalz, M. Ballauff and A. H. E. Muller, Nat. Mater., 2008, 7, 718.

35 R. Erhardt, A. Böker, H. Zettl, H. Kaya, W. Pyckhout-Hintzen, G. Krausch, V. Abetz and A. H. E. Muller, Macromolecules, 2001, 34(4), 1069.

36 A. Adedeji, S. Lyu and C. W. Macosko, Macromolecules, 2001, 34(25), 8663.

37 G. He, V. V. Ginzburg and A. C. Balazs, J. Polym. Sci., Part B: Polym. Phys., 2006, 44(17), 2389. 
38 V. V. Ginzburg, Macromolecules, 2005, 38(6), 2362.

39 J. J. Chiu, B. J. Kim, G.-R. Yi, J. Bang, E. J. Kramer and D. J. Pine, Macromolecules, 2007, 40(9), 3361.

40 B. J. Kim, J. J. Chiu, G.-R. Yi, D. J. Pine and E. J. Kramer, Adv. Mater., 2005, 17(21), 2618.

41 L. Zeyu, B. Haoab and Y. Zhang, RSC Adv., 2015, 5, 40668. 42 Y. Abe and G. Takahiro, Prog. Polym. Sci., 2004, 29(3), 149.

43 Y. Mai and A. Eisenberg, Chem. Soc. Rev., 2012, 41, 5969.

44 J. N. Israelachvili, Intermolecular and Surface Forces, Elsevier, Amsterdam, 2011.

45 C. Fong, T. Le and C. J. Drummond, Chem. Soc. Rev., 2012, 41, 1297.

46 M. L. Adams, A. Lavasanifar and G. S. Kwon, J. Pharm. Sci., 2003, 92, 1343.

47 A. Halperin and S. Alexander, Macromolecules, 1989, 22, 2403.

48 I. M. Davletbaeva, A. I. Akhmetshina, R. S. Davletbaev, I. I. Zaripov, A. M. Gumerov and R. R. Sharifullin, Polym. Sci., Ser. B, 2014, 56(6), 781.

49 R. Davletbaev, A. Akhmetshina, A. Gumerov, I. Davletbaeva and V. Parfenov, Composite Interfaces Special Issue: The
Fourth Asia Symposium on Advanced Materials (ASAM-4), 2014, vol. 7, p.611.

50 I. M. Davletbaeva, V. F. Shkodich, A. M. Gumerov, O. Yu. Emelina and A. V. Naumov, Polym. Sci., Ser. A, 2010, 52(4), 392.

51 A. I. Akhmetshina, R. S. Davletbaev, I. M. Davletbaeva, A. V. Mikhailova, A. M. Gumerov and R. Ya. Deberdeev, Russ. J. Appl. Chem., 2015, 88(3), 494.

52 I. M. Davletbaeva, G. R. Nurgaliyeva, A. I. Akhmetshina, R. S. Davletbaev, A. A. Atlaskin, T. S. Sazanova, S. V. Efimov, V. V. Klochkov and I. V. Vorotyntsev, RSC $A d v .$, 2016, 6, 111109.

53 I. M. Davletbaeva, A. I. Mazil'nikov, I. I. Zaripov, R. S. Davletbaev, A. M. Gumerov and V. V. Parfenov, Polym. Sci., Ser. B, 2018, 60(1), 51.

54 I. M. Davletbaeva, I. I. Zaripov, A. I. Mazilnikov, R. S. Davletbaev, R. R. Sharifullin, A. A. Atlaskin, T. S. Sazanova and I. V. Vorotyntsev, Membranes, 2019, $9(3), 42$. 